\title{
La paradoja del niño o niña: aplicaciones para la clase de probabilidad
}

\author{
José M. Contreras \\ jmcontreras@ugr.es \\ Universidad de Granada \\ España
}

\author{
Carmen Batanero \\ batanero@ugr.es \\ Universidad de Granada \\ España
}

\author{
Gustavo Cañadas \\ grcanadas@ugr.es \\ Universidad de Granada
}

España

\author{
Pedro Arteaga \\ parteaga@ugr.es \\ Universidad de Granada \\ España
}

Recibido: 1 Enero, $2013 \quad$ Aceptado: 20 Mayo 2013

Resumen. En la historia de la probabilidad encontramos diferentes paradojas que permiten al profesor organizar actividades didácticas en la enseñanza y el aprendizaje. Como ejemplo, en este trabajo analizamos la paradoja del niño o niña, su historia, algunas variantes, soluciones, objetos matemáticos trabajados y dificultades de los estudiantes, tales como la sesgo de equiprobabilidad y confusión entre probabilidad condicional y conjunta.

Palabras clave: Probabilidad, probabilidad condicional, paradojas, aplicaciones docentes

Abstract. In the history of probability there are various paradoxes which aid in organizing teaching and learning activities. As an example, in this paper we analyze the Boy and Girl paradox, its history, several variations, solutions, and the mathematical objects involved and students? potential difficulties, such as the equiprobability bias and confusion between joint and conditional probability.

KeyWords: Probability, conditional probability, paradoxes, didactic applications

\subsection{Introducción}

Aunque la probabilidad se incluye en la educación secundaria desde hace más de veinte años, en las orientaciones curriculares recientes (ej. NCTM, 2000; Ministerio de Educación y Ciencia (MEC), 2006) se propone un cambio metodológico con la finalidad de mejorar las intuiciones, que en el campo de la probabilidad, son, con frecuencia, erróneas. 
En trabajos anteriores (Contreras, Batanero, Arteaga y Cañadas, 2011 a y b) hemos sugerido el interés del uso de paradojas, como herramienta didáctica que permite enfrentar a los estudiantes con sus intuiciones incorrectas y hacerlas evolucionar de forma positiva, a la vez que aumenta su motivación. La historia de la probabilidad presenta situaciones muy atractivas que pueden conducir a reflexionar sobre la presencia del azar en la cotidianidad y son asequibles a los estudiantes. Por su carácter paradójico, enfrentan al alumno a una situación de resolución de un verdadero problema. El debate de las soluciones obtenidas por parte de los mismos estudiantes y la posterior aceptación de la solución correcta permiten también aplicar en aula las diferentes etapas en la teoría de situaciones didácticas propuesta por Brousseau (1986).

En este trabajo, describimos la paradoja del niño o niña (Gardner, 1959), analizada con frecuencia en la literatura sobre alfabetización estadística, presentando algunas variantes y soluciones, los contenidos matemáticos que se trabajan y posibles dificultades.

\subsection{Interés didáctico de las paradojas}

Son muchos los autores que defienden el interés de utilizar algunas paradojas sencillas en la clase de matemáticas, pues permiten plantear situaciones motivadoras en el aula, refuerzan la motivación y la meta-cognición de los estudiantes, y les hace descubrir conexiones entre la historia y la vida cotidiana. El uso inteligente de las mismas apoya una pedagogía constructivista, promoviendo un aprendizaje profundo, partiendo de las creencias previas (con frecuencia erróneas) que los alumnos pueden revisar al resolverlas (Lesser, 1998). A la vez, el análisis y discusión de las soluciones a las mismas exige al alumno una reflexión sobre sus propios procesos de pensamientos, lo que es tan importante como el aprendizaje de la solución correcta y un paso vital para alcanzar la capacidad matemática abstracta (Falk y Konold, 1992).

León (2009) indica que podemos servirnos de algunas de estas paradojas clásicas para crear situaciones didácticas que sirvan para provocar la reflexión didáctica de los alumnos. No es difícil encontrar este tipo de situaciones, pues la construcción de la teoría de la probabilidad no ha sido sencilla y soluciones incorrectas a problemas de probabilidad fueron a veces publicadas por matemáticos famosos (Batanero, Henry y Parzysz, 2005). Un proceso similar se desarrolla en el aprendizaje de los alumnos, pues el error puede convertirse en un factor de aprendizaje, siempre que se reconozca como tal por parte de los estudiantes, quienes deben construir su conocimiento mediante un proceso gradual (Borassi, 1987).

González (2004) señala que el uso de la historia con fines didácticos depende del conocimiento histórico del profesor y su iniciativa para adaptar este saber a los intereses y necesidades del grupo, por lo que ha de exponer los avances de la disciplina junto con su estado actual teórico y de aplicabilidad. El estudio de la historia de la probabilidad y de las paradojas asociadas a la misma será entonces un componente importante en la preparación de formadores.

El profesor que participa en estos cursos ya conoce los conceptos básicos de probabilidad, pero no siempre es consciente de las dificultades de los estudiantes o incluso de sus propias intuiciones incorrectas. El formador de profesores podría proponer algunos de estos problemas al comienzo del curso. La búsqueda de la solución correcta y el debate de las incorrectas, será un factor motivador, que lleve 
al profesor a interesarse por mejorar su formación sobre la didáctica de la probabilidad, a la vez que le lleva a reconocer sus propios sesgos de razonamiento.

\subsection{Historia de la paradoja}

Esta paradoja, también conocida como el problema de los dos hijos, es una variante de la conocida como paradoja de la caja de Bertrand y fue descrita por Gardner (1959), quien lo tituló los niños de Smith o el problema del señor Smith. El enunciado inicial de la paradoja se describió mediante dos opciones de la siguiente manera, haciendo el supuesto que los dos sexos son equiprobables:

- El Sr. Smith tiene dos hijos. El hijo mayor es una niña. ¿Cuál es la probabilidad de que ambos sean niñas?

- El Sr. Smith tiene dos hijos. Al menos uno de ellos es un niño. ¿Cuál es la probabilidad de que ambos hijos sean niños?

La primera solución que viene a la mente es pensar que la estructura de las dos preguntas es similar, dando la respuesta intuitiva $1 / 2$ para ambas. Esto es debido que la segunda pregunta lleva al lector a creer que los dos posibles casos para el sexo del segundo hijo (es decir, niño y niña) son equiprobables, y que la probabilidad de estos resultados es independiente de la información dada.

Gardner (1959) inicialmente dio como solución $1 / 2$ y 1/3, respectivamente, a las dos preguntas anteriores, pero más tarde reconoció que la segunda era ambigua, pues la respuesta podría ser también $1 / 2$, dependiendo de cómo se obtuvo la información de que uno de los hijos del Sr. Smith era un niño. Bar-Hillel y Falk (1982) y Nickerson (2004) confirmaron posteriormente la ambigüedad de la anterior formulación.

Esta paradoja ha estimulado una gran controversia. En primer lugar, el espacio muestral de todos los eventos posibles puede ser fácilmente enumerado: \{Niño-Niño, Niño-Niña, Niña-Niño, Niña-Niña\}. En segundo lugar, se supone que estos resultados son igualmente probables. Los supuestos son los siguientes:

1. Que cada niño es hombre o mujer.

2. El sexo de cada hijo es independiente del sexo del otro.

3. Que cada hijo tenga la misma probabilidad de ser hombre como de ser mujer.

Estos supuestos no son totalmente ciertos ya que la proporción de niños y niñas no es exactamente de 50:50, y (entre otros factores) la posibilidad de gemelos idénticos significa que la determinación del sexo no es totalmente independiente. Pero se asumen como verdaderos.

\subsection{Algunas soluciones correctas y objetos matemáticos que se trabajan}

Solución intuitiva correcta para la primera cuestión. En la primera cuestión, el Sr. Smith tiene dos hijos y el mayor es una niña; se nos pide la probabilidad de que ambos hijos sean niñas. En el espacio muestral del problema encontramos que hay cuatro eventos igualmente probables, pero que dos de ellos no 
cumplen la condición de que el hijo mayor sea una niña (Tabla 1.1).

Puesto que las dos posibilidades del espacio muestral reducido $\{$ Niña-Niño, Niña-Niña $\}$ son igualmente probables, y sólo uno de los dos incluye dos niñas, la probabilidad de que el hijo más pequeño sea también una niña es $1 / 2$. Aunque no es necesario, se podría usar el diagrama en árbol (Figura 1.1) para representar el problema. La condición supone que se elimina la rama superior.

Primer Hijo Segundo Hijo

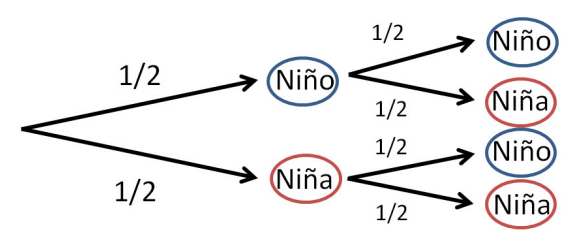

Figura 1.1: Posibles resultados del experimento

\begin{tabular}{|l|l|}
\hline Hijo mayor & Hijo menor \\
\hline Niña & Niña \\
\hline Niña & Niño \\
\hline Niño & Niña \\
\hline Niño & Niño \\
\hline
\end{tabular}

Tabla 1.1: Espacio muestral del problema

Solución intuitiva correcta para la segunda cuestión. En la segunda cuestión, se indica que el Sr. Smith tiene dos hijos y al menos uno de ellos es un niño y se pregunta la probabilidad de que ambos hijos sean niños. Aunque esta cuestión se parezca a la primera, la condición dada es diferente pues, en lugar de especificar el sexo del hijo mayor, se dice que al menos uno de los hijos es un niño.

$\mathrm{Al}$ considerar el espacio muestral inicial, como en el primer apartado, vemos que hay cuatro posibles eventos en el espacio muestral. Tres de estas familias reúnen la condición necesaria y suficiente de tener al menos un niño. El espacio muestral reducido se presenta en la Tabla 1.2, donde vemos que hay un caso favorable de tres. Una información fundamental para resolver el problema es cómo fue seleccionada la familia del Sr. Smith y cómo se obtuvo la información. Si no se sabe nada, se aceptan todos los sucesos posibles y la respuesta a la cuestión 2 sería 1/3.

\begin{tabular}{|l|l|}
\hline Hijo mayor & Hijo menor \\
\hline Niña & Niña \\
\hline Niña & Niño \\
\hline Niño & Niña \\
\hline Niño & Niño \\
\hline
\end{tabular}

Tabla 1.2: Espacio muestral de la variable aleatoria

Solución analítica correcta para la segunda cuestión. A partir de la regla del producto en el experimento compuesto, podemos calcular probabilidades a priori de los tres casos posibles:

1. Ambos son niñas (Niña-Niña), con probabilidad $P($ Niña-Niña $)=1 / 4$;

2. Ambos son varones (Niño-Niño), con probabilidad de $P($ Niño-Niño $)=1 / 4, \mathrm{y}$

3. Un hijo es niño y el otro niña, $O=\{$ Niña-Niño, Niño-Niña $\}$, con una probabilidad de $P(O)=1 / 2$. 
Sea $H$ el suceso "al menos uno es un niño". Aplicando el teorema de Bayes, tenemos:

$$
\begin{aligned}
P(\text { Niño-Niño } / H) & =\frac{P(\text { Niño-Niño } \cap H)}{P(H)} \\
& =\frac{P(H / \text { Niño-Niño }) \times P(\text { Niño-Niño })}{P(H)} \\
& =\frac{1 \times 1 / 4}{3 / 4}=1 / 3 .
\end{aligned}
$$

Un diagrama en árbol como el que se muestra en la Figura 1.2 podría facilitar la comprensión de todos los pasos en la solución del problema, ya que se identifica fácilmente la reducción del espacio muestral y las probabilidades condicionadas del suceso $H$ a cada una de los tipos de familias. Estas dos soluciones se pueden trabajar en el aula, dependiendo de los conocimientos de los estudiantes y el nivel educativo en el que estemos trabajando. En la Tabla 3 presentamos los objetos matemáticos que se trabajan, que pueden variar dependiendo de la solución dada.

\begin{tabular}{|c|c|c|c|c|}
\hline Tipo & Objetos matemáticos & Significado en el problema & Solución 1 & Solución 2 \\
\hline \multirow{7}{*}{ Lenguajes } & & & $\mathrm{x}$ & $\mathrm{x}$ \\
\hline & - Verbal & - Explicación de la situación & & \\
\hline & & & Opcional & Opcional \\
\hline & - Gráfico & - Diagrama en árbol & & \\
\hline & & & $\mathrm{x}$ & $X$ \\
\hline & - Simbólico & - Expresar sucesos, probabilidades & & \\
\hline & - Numérico & - Valor de la probabilidad & $\mathrm{x}$ & $X$ \\
\hline
\end{tabular}

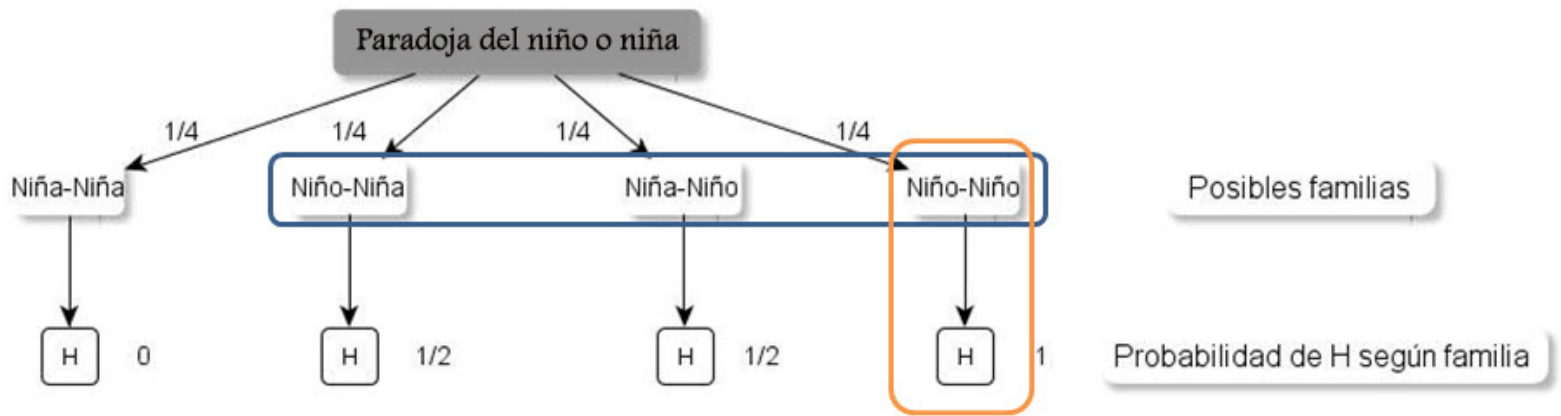

Figura 1.2: Posibles resultados del experimento 


\begin{tabular}{|c|c|c|c|c|}
\hline \multirow{7}{*}{ Conceptos } & - Experimento aleatorio & - Sexo del niño & $\mathrm{x}$ & $x$ \\
\hline & - Sucesos; espacio muestral & - Niño o niña & $\mathrm{x}$ & $X$ \\
\hline & - Experimento compuesto & - Sexo de dos niños & $\mathrm{x}$ & $\mathrm{x}$ \\
\hline & $\begin{array}{l}\text { - Sucesos en el experimento } \\
\text { compuesto }\end{array}$ & $\begin{array}{l}\text { - Niño-niño, niño-niña, niña-niño, } \\
\text { niña-niña }\end{array}$ & $\mathrm{x}$ & $\mathrm{x}$ \\
\hline & - Intersección de sucesos & - Conjunto común de sucesos. & $\mathrm{x}$ & $\mathrm{x}$ \\
\hline & - Probabilidad clásica & $\begin{array}{l}\text { - Proporción de casos favorables a } \\
\text { posibles. }\end{array}$ & $\mathrm{x}$ & $\mathrm{x}$ \\
\hline & - Probabilidad condicional & $\begin{array}{l}\text { - Proporción de ocurrencia suceso } \\
\text { dada una condición }\end{array}$ & $\mathrm{x}$ & $\mathrm{x}$ \\
\hline \multirow[t]{4}{*}{ Procedimientos } & $\begin{array}{l}\text { - Cálculo probabilidades } \\
\text { intuitivo }\end{array}$ & - Aplicar reglas intuitivas & $\mathrm{x}$ & \\
\hline & $\begin{array}{l}\text { - Cálculo probabilidades } \\
\text { formal }\end{array}$ & - Aplicar reglas de cálculo formal & & $\mathrm{x}$ \\
\hline & $\begin{array}{l}\text { - Reducción de espacio } \\
\text { muestral }\end{array}$ & - Aplicar condiciones & $\mathrm{x}$ & $\mathrm{x}$ \\
\hline & - Representación gráfica & - Diagrama; esquema & $\mathrm{x}$ & $x$ \\
\hline \multirow[t]{4}{*}{ Propiedades } & $\begin{array}{l}\text { - Diferencia probabilidad } \\
\text { condicionada y simple }\end{array}$ & - Restricción del espacio muestral & $\mathrm{x}$ & $x$ \\
\hline & - Regla del producto & - $P(A \cap R)=P(A) P(R / A)$ & & $x$ \\
\hline & $\begin{array}{l}\text { - Teorema probabilidad to- } \\
\text { tal }\end{array}$ & - Aplicar a la situación & & $x$ \\
\hline & - T. Bayes & - Aplicar a la situación & & $\mathrm{x}$ \\
\hline
\end{tabular}




\begin{tabular}{|l|l|l|l|l|}
\hline Argumento & • Razonamiento deductivo & $\bullet$ Demostración de la solución & $\mathrm{x}$ & $\mathrm{x}$ \\
\hline
\end{tabular}

Tabla 1.3: Objetos matemáticos implícitos en la solución

Observamos que el trabajo con este problema, es abordable en la educación secundaria o en la universidad, ya que es en estos niveles educativos donde se estudia la probabilidad condicional y el teorema de Bayes. También puede ser útil en cursos de formación de profesores, sobre todo si se realiza con los profesores el análisis de las diferentes soluciones posibles y de los objetos matemáticos involucrados. No sólo permite a los profesores trabajar con los objetos matemáticos ligados a la probabilidad condicional, sino mejorar su conocimiento didáctico. Por tanto, esta sencilla pero contra-intuitiva paradoja puede ser utilizada en el aula, ya que ilustra algunos principios básicos de la enseñanza de la teoría de probabilidades. Por supuesto es posible también un trabajo con mayor nivel de formalización, lo que implicaría un conjunto diferente de objetos matemáticos usados.

\subsubsection{Posibles dificultades a la hora de interpretar la paradoja}

Esta paradoja tiene valor pedagógico, ya que ilustra una aplicación interesante del comportamiento de la probabilidad condicional (Contreras, 2011). Fox y Levav (2004) utilizaron el problema para analizar como los alumnos estiman las probabilidades condicionales. El estudio encontró que el 85\% de los participantes respondieron correctamente al primer problema, mientras que sólo el 39\% respondió correctamente a la segunda pregunta. Los autores alegaron que la razón se debe a una utilización ingenua de la heurística de equiprobabilidad, ya que no se define exactamente el número de resultados posibles. También puede deberse a confusión entre probabilidad condicional y conjunta.

Otro razonamiento erróneo es suponer que la familia fuese seleccionada al azar entre todas las familias de dos hijos y se pidiese calcular la probabilidad de que los dos hijos fuesen niños, sin tener en cuenta la información de que uno de los hijos es niño. Como observamos en la Figura 1, la probabilidad de que el primer hijo fuese niño y el segundo también lo sea es de $1 / 4$, ya que:

$$
P\left(1^{o} \mathrm{Niño} \cap 2^{o} \mathrm{Niño}\right)=P\left(2^{o} \mathrm{Niño} / 1^{\circ} \mathrm{Niño}\right) \times P\left(1^{\circ} \mathrm{Niño}\right)=\frac{1}{2} \cdot \frac{1}{2}=\frac{1}{4}
$$

La paradoja surge porque el segundo enunciado (al menos uno de los dos hijos de una familia con dos hijos es niño) es difícil de comprender, y podría llevar a contradicciones si utilizamos el teorema de Bayes para su resolución. Por ejemplo, una interpretación posible es suponer que se trata de un problema de muestreo sin reposición, donde hemos tomado un elemento (un niño) y deseamos averiguar la probabilidad de que el segundo también sea niño.

Se haría el siguiente razonamiento. Sea $o$ el suceso "el elemento obtenido en la muestra es un niño". Entonces:

$$
\begin{aligned}
P((\text { Niño-Niño }) / o) & =\frac{P((\text { Niño-Niño }) \cap o)}{P(o)} \\
& =\frac{P(o /(\text { Niño-Niño })) \times P(\text { Niño-Niño })}{P(o)}=\frac{1 \times 1 / 4}{1 / 2}=1 / 2 .
\end{aligned}
$$

La diferencia de este razonamiento con la solución dada anteriormente, es que aquí $\mathrm{P}(0)$ es la probabilidad simple de seleccionar un niño al azar que es 0,5 , mientras el enunciado indica "al menos un niño en una muestra de dos". Es decir en este caso se puede tener un niño o dos niños. 
Esta paradoja es un ejemplo de cómo los enunciados de problemas relacionados con la probabilidad condicional pueden inducir a errores de interpretación. Pollatsek, Well, Konold y Hardiman (1987) coinciden en que muchas de las dificultades que las personas tienen con la comprensión de la probabilidad condicional pueden deberse a la redacción de los enunciados. Su hipótesis se basa en los resultados de Einhorn y Hogarth (1986), quienes sugieren que los enunciados que usan la conjunción " $y$ " pueden llevar a confundir la probabilidad conjunta y la probabilidad condicional.

\subsection{Variantes de la paradoja}

Paradoja del Señor Smith. A raíz de la popularización de la paradoja de Gardner se presentaron y discutieron diferentes variantes. La primera de ellas, Bar-Hillel y Falk (1982), tiene el siguiente enunciado:

El Sr. Smith es el padre de dos hijos. Nos encontramos con él caminando por la calle con un niño, a quien se enorgullece en presentar como su hijo. ¿Cuál es la probabilidad de que otro hijo del Sr. Smith sea también un niño?

Bar-Hillel y Falk (1982) utilizan esta variante para resaltar la importancia de considerar los supuestos subyacentes. La respuesta intuitiva es $1 / 2$ y, cuando se hacen las suposiciones más naturales, esto es correcto. Sin embargo, alguien puede argumentar que “... antes de que el Sr. Smith identifica al niño como su hijo, sólo sabemos que él es o bien el padre de dos niños (Niño-Niño), o de dos niñas(NiñaNiña), o de uno de cada sexo en cualquiera orden de nacimiento(Niña-Niño,Niño-Niña)". Suponiendo de nuevo independencia y equiprobabilidad, tenemos una probabilidad de $1 / 4$ de que Smith es el padre de dos niños. Al descubrir que él tiene por lo menos un niño, descartamos el suceso Niña-Niña. Dado que los restantes tres eventos son equiprobables, se obtiene una probabilidad de 1/3 para Niño-Niño.

Paradoja de los cachorros beagles. Otras variantes de esta paradoja fue popularizada por Savant (1991, 1996). La primera versión del problema planteado por Savant tiene el siguiente planteamiento:

Un tendero dice que tiene dos cachorros beagles, pero que no sabe si son machos, hembras o parejas. Usted le dice que quiere un macho, y el tendero llama por teléfono a un compañero que les está dando un baño. ¿Hay por lo menos un macho? ¡Sí! se le informa el compañero. ¿Cuál es la probabilidad de que el otro sea un macho?

Savant (1991) dio como respuesta "uno de cada tres". Defendió su conclusión, observando que hay cuatro parejas posibles en tres de las cuales al menos uno es macho, pero sólo en una ambos son machos.

La confusión de algunas personas que piensan que la respuesta es $1 / 2$ surge porque al compañero del tendero no se le pregunta si el cachorro al que está lavando es un macho, sino que se le pregunta si alguno de ellos es macho. El orden de nacimiento de los cachorros es irrelevante. Si se pudiera diferenciar el orden; por ejemplo, si los cachorros estuvieran etiquetados ( $A$ y $B$ ), cada uno tendría una probabilidad del $50 \%$ de ser macho, independientemente del sexo del otro. Esta independencia se restringe cuando se establece que al menos uno de los dos $A$ o $B$ es macho. Con dicha información, si $A$ no es macho, $B$ debe serlo y viceversa. Esta restricción se introduce por la forma en que está estructurada la pregunta y se pasa por alto fácilmente, llevando a la respuesta errónea del $50 \%$. 
Debido al gran número de conclusiones erróneas que la autora recibió, Savant (1996) vuelve al plantear el problema de forma diferente:

Un cliente y un tendero, que no están relacionados, tienen dos hijos cada uno. Sabemos que por lo menos uno de los hijos del tendero es un niño y que el hijo mayor del cliente es un niño. ¿Puede explicar por qué las probabilidades de que el tendero tenga dos niños no son iguales a las probabilidades de que el cliente tenga dos niños?

Siguiendo razonamientos similares a los ya expuestos, Savant concluye que la probabilidad de que el tendero tenga dos niños es $1 / 3$, mientras que la probabilidad de que el cliente tenga dos niños es $1 / 2$; en el primer caso no se dice cuál de los hijos es el niño, mientras que en el segundo se dice que es el mayor. Para corroborar los resultados el autor realizó una encuesta entre sus lectores, pidió a aquellos con exactamente dos hijos, de los cuáles al menos uno fuese niño le escribiesen indicando el sexo de ambos hijos. Con casi 18000 respuestas, los resultados mostraron que un 35,9\% de los que cumplían la condición tenían dos niños. Carlton y Stansfield (2005) afirman que los resultados de esta encuesta validan empíricamente las soluciones de Savant a este problema; las probabilidades de que el tendero y el cliente tengan dos niños son diferentes y en el caso del tendero, esta probabilidad es más cercana de 1 de cada 3 que a 1 de cada 2 .

Problema del nacimiento en martes. La siguiente versión de la paradoja fue descrita por Foshee (2010) en un congreso en homenaje a Gardner.

Supongamos que nos dijeron no sólo que el señor Smith tiene dos hijos, y uno de ellos es un niño, sino también que el niño nació en un martes ¿cuál es la probabilidad de que haya dos niños?

La cuestión que nos planteamos es la siguiente: ¿Cambia esto nuestros análisis anteriores? ¿Si tiene dos hijos, y uno es un niño, entonces la probabilidad de tener dos hijos es significativamente diferente si se proporciona la información adicional de que el niño nació en un martes? Una solución intuitiva a este problema sería contar las diferentes combinaciones de géneros y días de la semana, y calcular el resultado aplicando la ley de Laplace, que da el valor 13/27:

(número de combinaciones con dos niños de los que al menos uno nació un martes)

(número de combinaciones con al menos un niño nacido en martes)

La explicación es la siguiente: supongamos que tenemos una población de familias con dos hijos, siendo el sexo de los dos hijos independiente, niño o niña igualmente probables, y que el día que nace cada hijo es independiente del día que nace el otro; cada día de la semana tiene la misma probabilidad 1/7. En la Figura 1.3 se muestran todas las parejas posibles de hijo, teniendo en cuenta su sexo así como los días de la semana posibles en los que se puede nacer (X significa Miércoles). El verde representa el suceso \{dos niños, al menos uno de los cuales nació un martes\}. El rojo representa el suceso \{al menos un niño nacido en martes\}. Por lo tanto la respuesta es:

$$
P=\frac{\text { verde }}{\text { verde }+ \text { rojo }}=\frac{13}{13+14}=\frac{13}{27} .
$$



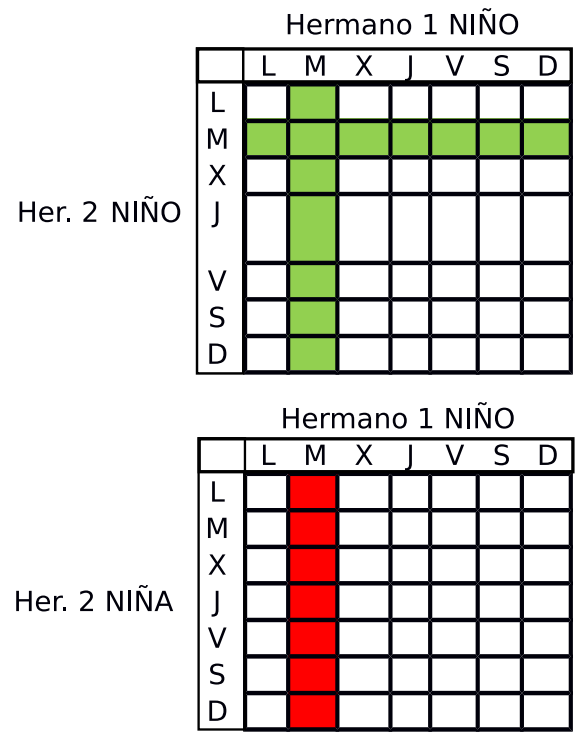
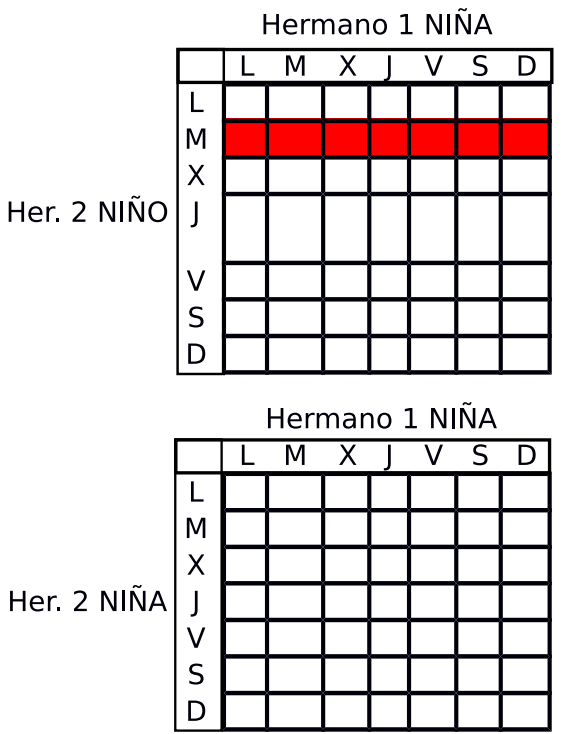

Figura 1.3: Posibles resultados del experimento

Nuestra intuición sugiere que los niños nacidos en martes no tienen mayor probabilidad de tener un hermano que los niños nacidos en otros días. Por lo tanto, el resultado 13/27 parece absurdo porque podríamos repetir con todos los días de la semana y llegar a la conclusión de que la respuesta al problema original es $13 / 27$ y no 1/3. La paradoja aparece porque en realidad no estamos calculando lo que pensamos. El problema no es "mi primogénito es un niño nacido en martes" o "tengo exactamente un niño nacido en martes", sino "uno, o más de uno, es un niño nacido en martes". El error viene del hecho de pensar que se ha partido el espacio muestral ( 2 géneros, 7 días de la semana) en 7 partes iguales.

La moraleja de la historia es que estas probabilidades no sólo dependen de la información que tenemos delante de nosotros, sino de cómo llegamos a esa información.

La paradoja de los cuatro hijos. Otra versión es una adaptación de la primera parte del primer problema de Gardner (1951), suponiendo cuatro hijos en la familia. El enunciado es el siguiente:

Supongamos que un matrimonio tiene cuatro hijos. ¿Cuál es la probabilidad de que dos de ellos sean niñas y dos niños?

Si suponemos como en las variantes anteriores la equiprobabilidad del nacimiento de varones y de mujeres, el sentido común nos impulsa a creer que en un caso como este la familia tendrá dos hijos y dos hijas o pensamos que existe una alta probabilidad de que dos de ellos serán niños, y dos niñas. La forma de abordar este problema es simple, bastaría con calcular el conjunto de posibles combinaciones, ver tabla 1.4, del espacio muestral "tener cuatro hijos", teniendo en cuenta el orden de nacimiento, siendo $O$ el suceso "ser niño" y $A$ el suceso "ser niña". 


\begin{tabular}{|l|l|}
\hline 1 & OOOO \\
\hline 2 & OOOA \\
\hline 3 & OOAO \\
\hline 4 & OOAA \\
\hline 5 & OAOO \\
\hline 6 & OAOA \\
\hline 7 & OAAO \\
\hline 8 & OAAA \\
\hline 9 & $A O O O$ \\
\hline 10 & $A O O A$ \\
\hline 11 & $A O A O$ \\
\hline 12 & $A O A A$ \\
\hline 13 & $A A O O$ \\
\hline 14 & $A A O A$ \\
\hline 15 & $A A A O$ \\
\hline 16 & $A A A A$ \\
\hline
\end{tabular}

Tabla 1.4: Espacio muestral paradoja de los cuatro hijos

Dado que solo hay dos sexos posibles, la cantidad de combinaciones existente para cuatro nacimientos son las 16 que se ven en la tabla anterior. Recordemos que todo nuestro análisis es válido porque estamos considerando que la probabilidad de que sea niño es igual a la de que sea niña ( $50 \%$ cada uno). Como indicamos anteriormente, en el mundo real dicha proporción no es exacta, pero se aproxima lo suficiente como para que los resultados que vamos a mostrar prácticamente no varíen.

El paso siguiente consiste en contar cada uno de los casos mostrados en la tabla. Vemos que, de los 16, solo hay dos casos en que el sexo de todos los hijos es el mismo (el 1 y el 16). Eso significa que tenemos una probabilidad de 2/16 de que nuestros cuatro hijos tengan el mismo sexo. Si contamos los casos en que los nacimientos incluyen un hijo de un sexo y tres del otro, encontramos ocho casos (en las filas $2,3,5,8,9,12,14$ y 15). Eso implica que en la mitad de los casos, un matrimonio que tenga cuatro hijos tendrá o bien una niña y tres niños, o bien un niño y tres niñas. Por último, si contamos los casos que nos interesan, aquellos en que hay dos niños de cada sexo, vemos que solo los casos 4, 6, 7, 10, 11 y 13 cumplen con la condición "dos niños y dos niñas."

Esto demuestra que solo 6 de cada 16 veces se da realmente la situación que nuestro sentido común decía era la más probable. Es decir sólo el 37,5\% de las familias con cuatro hijos tendrá dos de cada sexo, y que es mucho más probable tener 3 hijos de un sexo y uno del otro que cualquiera de las otras posibilidades por separado.

\subsection{Conclusiones.}

En el artículo hemos presentado un ejemplo de una paradoja que permite introducir una situación de interés para el estudiante y motivarle en el estudio de la probabilidad. Además de trabajar diversos objetos matemáticos el alumno adquiere competencia para considerar con cuidado las condiciones de cada problema e identificar los sucesos de interés. 
Es necesario también advertir al profesor de las posibles dificultades de los estudiantes al resolver los problemas, como se ha mostrado en otras investigaciones que hicieron uso de paradojas en la formación de profesores. Así Contreras (2011) informa que en un taller en el que participaron 166 profesores en servicio y en formación, sólo el 24,7\% resolvió correctamente el problema en la presentación inicial de la paradoja de Bertrand (Contreras et al., 2011). Las soluciones incorrectas fueron debidas principalmente a confusiones entre probabilidad condicional y conjunta, heurística de representatividad (donde algunos profesores esperaban una convergencia muy exacta de las frecuencias de resultados a la probabilidad en pocos ensayos) y sesgo de equiprobabilidad.

La discusión colectiva y simulación de la situación llevó al 67\% de los participantes a descubrir la solución correcta al final de la experiencia. Pero aún así, el resto no llegó a cambiar su postura inicial; lo cual indica que sería necesario un mayor tiempo en la actividad o complementarla con otras similares.

Por otro lado, el uso de paradojas responde a un cambio metodológico en la enseñanza de la probabilidad, que implica la necesidad de una mayor formación del profesor, quien, además de la formación científica, requiere un "conocimiento profesional", en el cual Ball, Thames y Phelps (2005) incluyen cuatro componentes: conocimiento común del contenido, conocimiento especializado del contenido, conocimiento del contenido y la enseñanza y conocimiento del contenido y los estudiantes. Por ello es también importante buscar actividades adecuadas para llevar a cabo esta formación. En particular, estas situaciones deberían permitir la reflexión epistemológica sobre la estadística, el estudio de las investigaciones didácticas sobre errores y dificultades de aprendizaje, y el análisis y experimentación de métodos y recursos de enseñanza.

Una de estas actividades pueden ser paradojas como la presentada en este trabajo, que, junto con las actividades de análisis didáctico pueden contribuir a la adquisición de los diferentes componentes del conocimiento profesional, en el caso de la probabilidad. Asimismo ayuda a mejorar la formación del profesor para llevar a cabo estos tipos de análisis, que puede aplicar al diseño de secuencias de enseñanza y el análisis reflexivo sobre la propia práctica docente, con la finalidad de favorecer el aprendizaje de los estudiantes.

Agradecimientos: Proyecto EDU2010-14947 (MCINN-FEDER) y al grupo FQM126 (Junta de Andalucía).

\section{Bibliografía}

[1] Batanero, C.; Henry, M.; Parzysz, B. The nature of chance and probability. In G. A. Jones (Ed.), Exploring probability in school: Challenges for teaching and learning (pp. 15-37). New York: Springer. 2006.

[2] Ball, D. L.; Thames, M. H.; Phelps, G. Articulating domains of mathematical knowledge for teaching. www-personal. umich.edu/ dball/, Consulta: 10/01/2013. 2005.

[3] Bar-Hillel, M.; Falk, R. Some teasers concerning conditional probabilities. Cognition, 11(2). 1992. 109-122.

[4] Borassi, R. Exploring mathematics through the analysis of errors. For the Learning of Mathematics,7 (3). 1987. 2-8.

[5] Brousseau, G. Fondements et Méthodes de la Didactique des Mathématiques. Recherches en Didactique des Mathématique, 7(2).1986. 33-115.

[6] Contreras, J. M. Evaluación de conocimientos y recursos didácticos en la formación de profesores sobre probabilidad condicional. Tesis doctoral. 2011. 
[7] Contreras, J.M.; Batanero, C.; Arteaga, P.; Cañadas, G. El dilema de los prisioneros: valor de las paradojas en la clase de matemáticas. Gamma: revista galega de educación matemática, 12.2011a. 31-37.

[8] Contreras, J.M.; Batanero, C.; Arteaga, P.; Cañadas, G. La paradoja de la caja de Bertrand: algunas formulaciones y cuestiones didácticas. Epsilon, 28(2).2011b.7-20.

[9] Einhorn, H. J.; Hogart, R. M. Judging probable cause. Psychological Bulletin, 99.1986. 3-19.

[10] Falk, R.; Konold, C. The pshycology of learning probability. En F. Gordon y S. Gordon (Eds.), Statistics for the twuenty-first century, MAA Notes 26 (pp. 151-164). Washington, DC: Mathematical Association of America. 1992.

[11] Fox C. R.; Levav J. Partition-edit-count: naive extensional reasoning in judgment of conditional probability. Journal of Experimental Psychology, 133. 2004. 626-642.

[12] Foshee, G. The Tuesday Birthday Problem. The ninth Gathering 4 Gardner. Atlanta. 2010.

[13] Gardner, M. Scientific American Book of Mathematical Puzzles and Diversions. Simon \& Schuster. 1959.

[14] Gardner, M. The Second Scientific American Book of Mathematical Puzzles and Diversions. Simon \& Schuster. 1961.

[15] González, P. La historia de la matemática como recurso didáctico e instrumento para enriquecer culturalmente su enseñanza. SUMA, 45. 2004. 17-28.

[16] Grinstead, C. M.; Snell, J. L. Grinstead and Snell's Introduction to Probability. The CHANCE Project. GNU Free DocumentationLicense. 2006.

[17] León, N. La historia como elemento motivador hacia el estudio de la probabilidad: el problema de la apuesta interrumpida, Sapiens: Revista Universitaria de Investigación, 1.2009. 69-88.

[18] Lesser, L.Countering indifference - Using counterintuitive example. TeachingStatistics, 20(1), 1998. 10-12.

[19] MEC. Real Decreto 1631/2006, de 29 de diciembre, por el que se establecen las enseñanzas mínimas correspondientes a la Educación Secundaria Obligatoria. 2006.

[20] NCTM. Principles and standards for school mathematics. Reston, VA; NCTM. standards.nctm.org/, Consulta: 10/01/2013. 2000.

[21] Savant, M. Parade Magazine. Númerode Octubre.1991.

[22] Savant, M. Parade Magazine. Número de Mayo. 1996.

[23] Nickerson, R. S.Cognition and Chance: The Psychology of Probabilistic Reasoning. Mahwah, NJ: Lawrence Erlbaum Associates Inc. 2004.

[24] Pollatsek, A.; Well, A. D.; Konold, C.; Hardiman, P. Understanding conditional probabilities. Organitation, Behavior and Human Decision Processes, 40, 255-269.1987. 